Original article

\title{
Directional functional coupling during limbic seizures in rats revealed by nonlinear Granger causality
}

\author{
Ilya V. Sysoev ${ }^{1,2}$, Martin F.J. Perescis ${ }^{3,4}$, Lyudmila V. Vinogradova ${ }^{5}$, Marina V. Sysoeva ${ }^{6}$, Clementina M. van Rijn ${ }^{3}$ \\ ${ }^{1}$ Saratov State University, Saratov, Russia \\ ${ }^{2}$ Saratov Branch of Kotel'nikov Institute of Radio Engineering and Electronics, Saratov, Russia \\ ${ }^{3}$ Donders Centre for Cognition, Radboud University, Nijmegen, Netherlands \\ ${ }^{4}$ HAS University Of Applied Sciences, 's-Hertogenbosch, Netherlands \\ ${ }^{5}$ Institute of Higher Nervous Activity and Neurophysiology, Moscow, Russia \\ ${ }^{6}$ Yuri Gagarin State Technical University of Saratov, Saratov, Russia
}

Received 30 August 2018, Revised 11 October 2018, Accepted 15 October 2018

(C) 2018, Sysoev I.V., Perescis M.F.J., Vinogradova L.V., Sysoeva M.V., van Rijn C.M.

(C) 2018, Russian Open Medical Journal

Abstract: Subject and Purpose - Temporal lobe epilepsy is increasingly recognized to involve widespread network alterations. In this study the temporal dynamics of directional interactions between the frontal cortex, hippocampus, thalamus and midbrain during limbic seizures are analyzed in rats.

Methods - Local field potentials were recorded in Wistar rats expressing generalized limbic seizures, Racine's stage 4-5. These spontaneous seizures occur during chronic treatment with a CB1 (cannabinoid receptor type 1) antagonist SLV326. Time-frequency analysis, time-varying adapted nonlinear Granger causality and mutual information were applied. The Granger causality and mutual information values estimated from seizure episodes were compared to those of seizure-free periods.

Results - During the seizure, two stages were detected: a high frequency $(15-20 \mathrm{~Hz})$ stage followed by a low frequency $(2 \mathrm{~Hz})$ stage. At seizure onset, a drop in coupling between all recorded sites was found. After the seizure onset, coupling restored to normal levels in the midbrain - hippocampus pair, but remained reduced in all other studied channel pairs for at least $10 \mathrm{~s}$ after the onset. The transition between stages and the seizure termination were characterized by couplings increase in some pairs and by decrease in others.

Conclusion - Spontaneous generalized limbic seizures can be considered as a result of pathological reorganization of coupling architecture between different brain structures, developing in time, and providing transitions between seizure stages. These findings bring together views, considering seizures as an increase of couplings in the brain, and hypotheses, regarding seizures rather as a decoupled state.

Keywords: temporal lobe epilepsy, limbic system, generalized seizures, Granger causality, seizure stages.

Cite as Sysoev IV, Perescis MFJ, Vinogradova LV, Sysoeva MV, van Rijn CM. Directional functional coupling during limbic seizures in rats revealed by nonlinear Granger causality. Russian Open Medical Journal 2018; 7: e0404.

Correspondence to llya V. Sysoev. Work address: Astrakhanskaya str., 83, Saratov, 410012, Russia. E-mail: ivssci@gmail.com. Tel.: +7 9063027189. Fax: +78452522705 .

\section{Introduction}

Spontaneous seizures involving the hippocampus and parahippocampal structures are the hallmark of temporal lobe epilepsy. Epileptic activity may propagate widely along synaptic pathways and can become highly synchronized between limbic and other regions [1, 2]. In the most widely used experimental model of temporal lobe epilepsy, spontaneous limbic seizures develop after an initial pharmacologically or electrically induced status epilepticus [3]. However, in non-epileptic rats, limbic seizures may also occur during chronic exposure to antagonists of cannabinoid receptor type 1 (CB1) [4].

The endocannabinoid system participates in the regulation of brain sensitivity to epileptic activation $[5,6]$. In the pilocarpine rat model for epileptogenesis $C B$ agonists reduce early post-status epilepticus seizure manifestations and subsequent mortality [7]. Moreover, in the same model, agonists abolish the occurrence of late spontaneous epileptic seizures [5]. CB1 antagonists on the other hand diminish the brain resistance to seizures [5]. It was found that they facilitate the spreading of seizures from the midbrain to limbic structures in rats prone to midbrain-driven audiogenic seizures [8]. What is more, healthy rats treated long term with $\mathrm{CB}$ antagonist become prone to spontaneous seizures [4]. These seizures are topic of the present paper.

Endocannabinoids are synthesized on demand after activation of postsynaptic neurons and they retrogradely suppress neurotransmitter release through presynaptic metabotropic CB1 receptors. In this way, endocannabinoids contribute to maintaining the physiological level of neuronal excitability and synaptic function [9]. Epileptic excitation quickly activates this defense mechanism against overexcitation [10]. Endocanabinoid CB1 receptors $[11,12]$ play the critical role in the endocannabinoid-dependent protection against seizures, and local deletion of these receptors in the hippocampus strongly exacerbates seizures [13]. 
Widespread extralimbic regions, including both cortical and subcortical structures, are increasingly recognized to be involved in secondary generalization of limbic seizures in epileptic rats [14, 15] and complex partial seizures in patients with temporal lobe epilepsy (TLE) $[15,16]$. The present study was aimed to analyze directional functional coupling between the neocortex, hippocampus, thalamus and midbrain during ictal period of SLV326-induced limbic seizures. The hippocampus is particularly prone to epileptic excitation. The midbrain region contains triggering areas for certain seizure types, primarily for generalized tonic-clonic seizures [17-19]. The thalamus is an important brain site for seizure propagation and its connectivity with the hippocampus and cortex is altered in patients with TLE [15, 20, 21].

Establishing results of the study on time series analysis, it is important to use methods which are capable to resolve a number of issues: 1) nonlinearity, 2) coupling directionality, 3) cause of similarity between signals, including direct links, common source, driving mediated through some intermediate structure, or even simple random coincidence of oscillations [22]. In this study the time varying adapted nonlinear Granger causality method [23] was chosen. This technique takes into account features of experimental data: time scales, nonlinearity, effective dimension. Therefore, it has a good temporal resolution with fine sensitivity and specificity, as it was shown [24, 25], being mostly insensitive to mediated coupling [24]

\section{Material and Methods}

\subsection{Subjects and local field potential recording}

The seizures analyzed in the present study occur during chronic treatment with the CB1 antagonist SLV326, as it was reported previously in [26]. Since the method to elicit the seizures was reported extensively there, here it will be repeated only in short.

The study was performed in accordance with the guidelines of the European Community for the use of experimental animals and was approved by the ethical committee for animal studies (RUDEC2007-161).

The electroencephalographic (EEG) experiments were performed on $24 \mathrm{Crl}: \mathrm{WI}$ Wistar rats (Charles River Laboratories, Sulzfeld, Germany), aged 7 months. From an age of 8-9 weeks, SLV326 was administered daily by oral gavage, $2-3 \mathrm{mg} / \mathrm{kg}$, dissolved in a semi-solid solution. Solutions of SLV326 were provided by Solvay Pharmaceuticals (Weesp, Netherlands).

The 24 animals were surgically provided with two tripolar EEG electrodes (Plastics One MS-332/2-A) under complete isoflurane anesthesia. Electrodes were placed in the following brain structures (distances provided in $\mathrm{mm}$ from bregma: anterior, lateral, and depth): frontal cortex (FC): $+2,-2,-1$; hippocampus (HP): $-4.2,-3.6,-4.1$; thalamus (TH): $-2.6,-2.7,-7.3$; and midbrain (MB): -8.8, -1.7, -5.2. Ground and reference electrodes were placed over the cerebellum bilaterally, with reference electrode located on the side ipsilateral to the recording electrodes. Animals were allowed to recover for at least two weeks.

LFP and video recordings were made during 24 hours. Local field potential (LFP) signals were amplified, filtered between 1 and $100 \mathrm{~Hz}$ and digitalized at the sample rate equal to $512 \mathrm{~Hz}$ using the Windaq system (DATAQ Instruments, Akron, Ohio, USA).
In the 24 hour recording period, 6 out of 24 rats demonstrated generalized limbic seizures. In the present study, 30 seizures recorded in three rats were analyzed, because for these rats all electrodes demonstrated the appropriate signal level during the whole recording.

\subsection{Application of time-variant adapted nonlinear Granger causality to LFP data}

To analyze the coupling between four measured signals from different brain areas, the adapted nonlinear time varying Granger causality method was used 23 . The method is based on the construction of two predicting models: a univariate model, which predicts values from the series $\left\{x_{n}\right\}_{n=1}^{N}$ based on only itself with the average error (1), and a bivariate one, which uses also data from the series $\left\{y_{n}\right\}_{n=1}^{N}$ (its prediction error (2) is usually less). The prediction improvement (3) is a main coupling measure and lies in between 0 and 1 .

$$
\begin{gathered}
\varepsilon_{s}^{2}=\frac{1}{N} \sum_{n=1}^{N}\left(x_{n}^{\prime}-x_{n}\right)^{2} \\
\varepsilon_{j}^{2}=\frac{1}{N} \sum_{n=1}^{N}\left(x^{\prime \prime}{ }_{n}-x_{n}\right)^{2} \\
P I=1-\frac{\varepsilon_{j}^{2}}{\varepsilon_{s}^{2}}
\end{gathered}
$$

where $x_{n}$ is a measured value, $x_{n}^{\prime}$ is a corresponding value predicted with a univariate model, and $x_{n}^{\prime \prime}$ is a value predicted with a bivariate model.

Absolute values of $P I$ are usually not informative, as it was shown by Smirnov and Mokhov [27]. However, increase or decrease of $P I$ makes sense under the condition that the operator of the evolution of the considered systems did not change structurally (but its parameters could change [24]). Changes in coupling strength can be detected this way, if Granger causality is used in a moving time window [28]. Here, a time window of length $1 \mathrm{~s}$ (512 data points) was used with window overlap of $0.9 \mathrm{~s}$. The method parameters, including time scales were adjusted in accordance with recommendations and criteria developed in [25, 29, 30].

\subsection{Mutual information function in moving window}

The mutual information function $(M I)$ was calculated for the same channel pairs and in the same conditions as for the Granger causality, including time window length and shift, and averaging, by means of the method proposed in [31]. Note, that $M I$ is an undirected nonlinear measure of simultaneous similarity in time series. This means that it cannot show the direction of driving or reveal the reason of changes, as almost all simple measures such as cross-correlation (linear or nonlinear) or coherence. The $\mathrm{MI}$ was applied to scalar series. While there are fine modern works [32], showing the possibility to improve the measure performance significantly by using the vector series with non-uniform embedding, this demands too many data to be applied in a moving window. Due to the same reason, the transfer entropy $[33,34]$ was not used. 

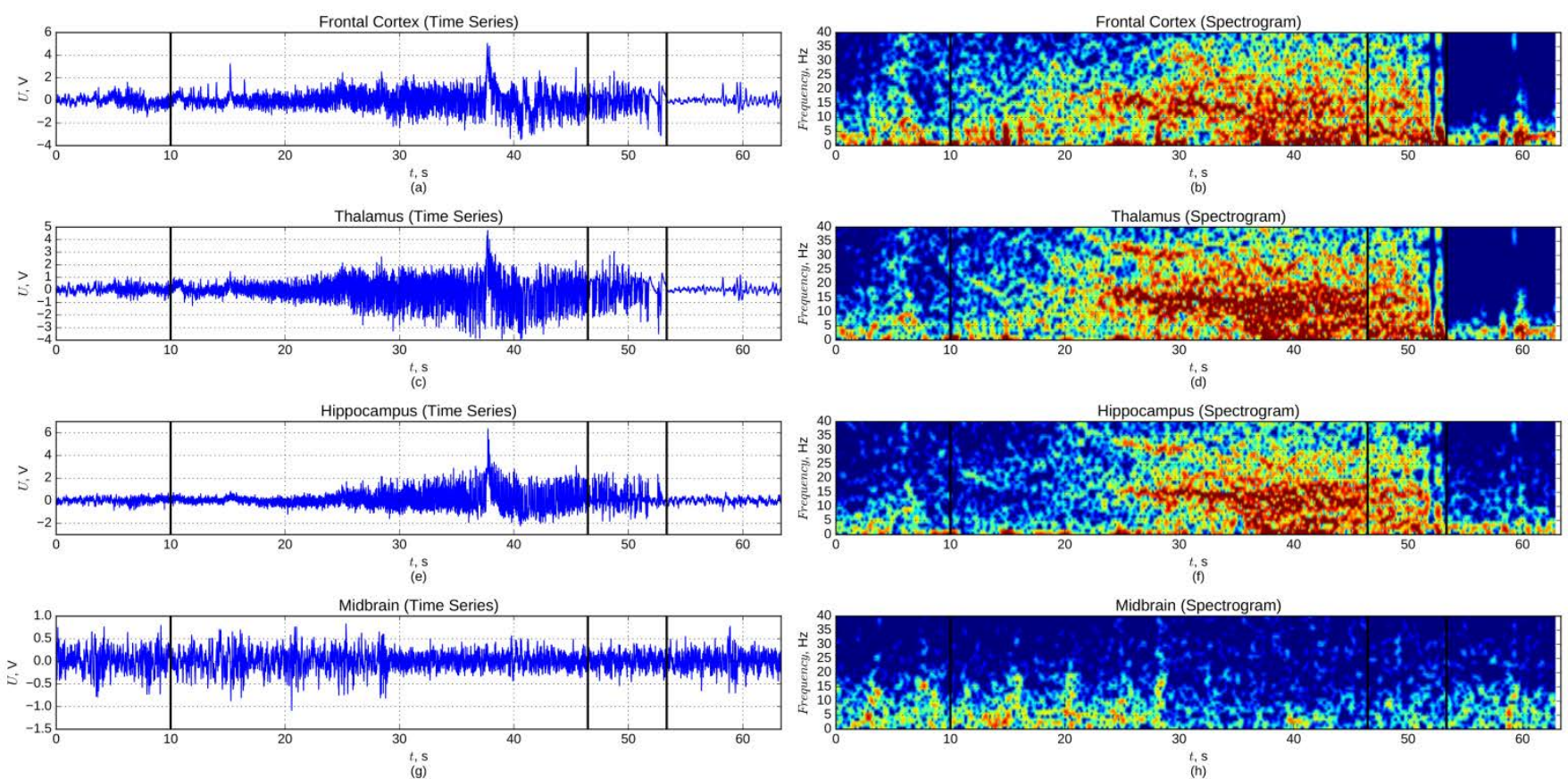

Figure 1. Local field potentials recorded during a limbic seizure and their spectrograms: (a, b) - from frontal cortex, (c, d) - from thalamus, (e, f) - from hippocampus, $(\mathrm{g}, \mathrm{h})$ - from midbrain. Three black vertical lines mark: 1) start of seizure at time point $10 \mathrm{~s}, 2)$ margin between two stages at time point $38 \mathrm{~s}$, and 3) end of seizure at $50 \mathrm{~s}$.

\subsection{Statistical analysis of coupling estimates}

For every seizure the individual dependencies $P I(t)$ and $M I(t)$ were calculated, including $10 \mathrm{~s}$ before and $10 \mathrm{~s}$ after the seizure. Accordingly, each considered EEG fragment containing a limbic seizure was split into three parts:

i) Initial: $10 \mathrm{~s}$ prior to and $10 \mathrm{~s}$ after seizure onset: preictal period and $10 \mathrm{~s}$ of the first high frequency stage;

ii) Middle: $10 \mathrm{~s}$ prior and $10 \mathrm{~s}$ after the transition from the first high frequency stage to the second low frequency stage;

iii) Final: $10 \mathrm{~s}$ before and $10 \mathrm{~s}$ after the seizure termination: low frequency stage and postictal period.

The resulting dependencies $P I(t)$ and $M I(t)$ were averaged across all seizures, matching onset, start of high frequency stage and seizure termination. Then, for each averaged dependency $P I(t)$ and $M I(t)$, the background levels $P I_{b g}$ and $M I_{b g}$, respectively, were established as an average value over the $3 \mathrm{~s}$ time interval (baseline period, from 10 to $7 \mathrm{~s}$ before the seizure onset). Using the mean baseline levels, the normalized dependencies were calculated as $P I_{O}(t)=P I(t)-P I_{b g}$ and $M I_{O}(t)=M I(t)-M I_{b g}$. The value of $P I_{O}=0$ (and $M I_{0}=0$ ) corresponds to the baseline level; positive values of $P I_{0}$ and $M I_{0}$ correspond to a larger coupling than in baseline and negative - to a lower one.

The values of $P I(t)$ and $M I(t)$ obtained for different seizures for the same time point were used as a sampling, and a single sampling t-test was performed to identify the statistical difference of the mean value from $\mathrm{Pl}_{b g}$ or $\mathrm{MI}_{b g}$ respectively. Since this test was performed for every time point, a Bonferroni-like correction for multiple testing was implemented: the achieved $p$-values were multiplied by a number of independent time intervals, from which $\mathrm{Pl}$ or $\mathrm{Ml}$ were calculated. If after correction a resulting $\mathrm{p}$-value occurred to be less than 0.05 , the results were considered as significantly different from baseline and plotted in color (blue if $P P<P l_{b g}$ or $M l<M l_{b g}$ and red if $P I>P I_{b g}$ or $M I>M I_{b g}$ ) in Figures 2 and 3. Otherwise, the results were plotted in gray as insignificant.

\section{Results}

\subsection{Time-frequency analysis}

All seizures started suddenly, with a sharp spike of small or medium amplitude detected in all recorded channels. The total duration of the seizures was from $35.0 \mathrm{~s}$ to $115.3 \mathrm{~s}$ with the mean duration of $53.1 \mathrm{~s}$. Two main stages can be distinguished (Figure 1):

i) a high frequency stage, starting from a frequency of about $20 \mathrm{~Hz}$ and decreasing to $15 \mathrm{~Hz}$ in the first $5 \mathrm{~s}$;

ii) a low frequency stage, at which the main seizure frequency dramatically falls to $2 \mathrm{~Hz}$.

The high frequency stage lasted for 23.3-55.7 s, with a mean duration of $36.0 \mathrm{~s}$. The amplitude of oscillations was rising during this stage. The whole stage was very nonstationary. Well pronounced higher frequencies can be seen in different time epochs.

This low frequency stage lasted for 5.7-59.6 s, with the mean longitude equal to $17.1 \mathrm{~s}$. The high frequency dynamics of the previous stage was also present in the first part of the second stage, albeit less pronounced. The low frequency oscillations were losing amplitude gradually and stopped suddenly. This power fall maked a very sharp marker of the seizure termination.

\subsection{Granger causality analysis}

Results of the coupling analysis, both using Granger causality and mutual information function are shown on Figures 2 and 3. 
(a)

Frontal Cortex-Midbrain

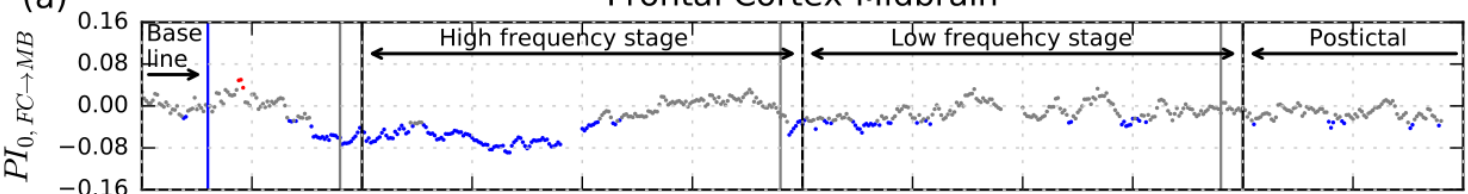

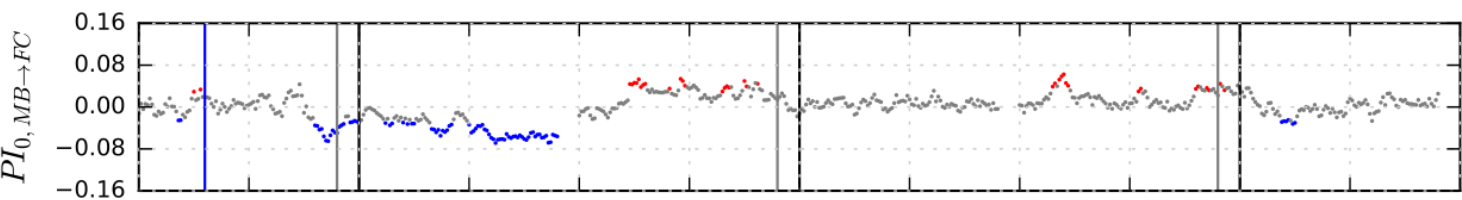

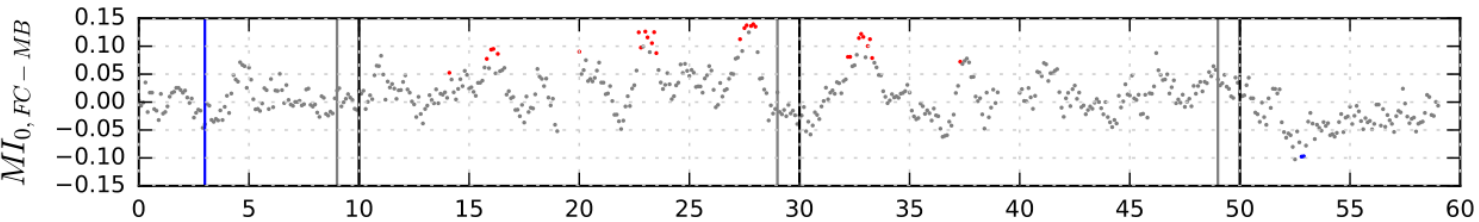

(b)

Frontal Cortex-Thalamus
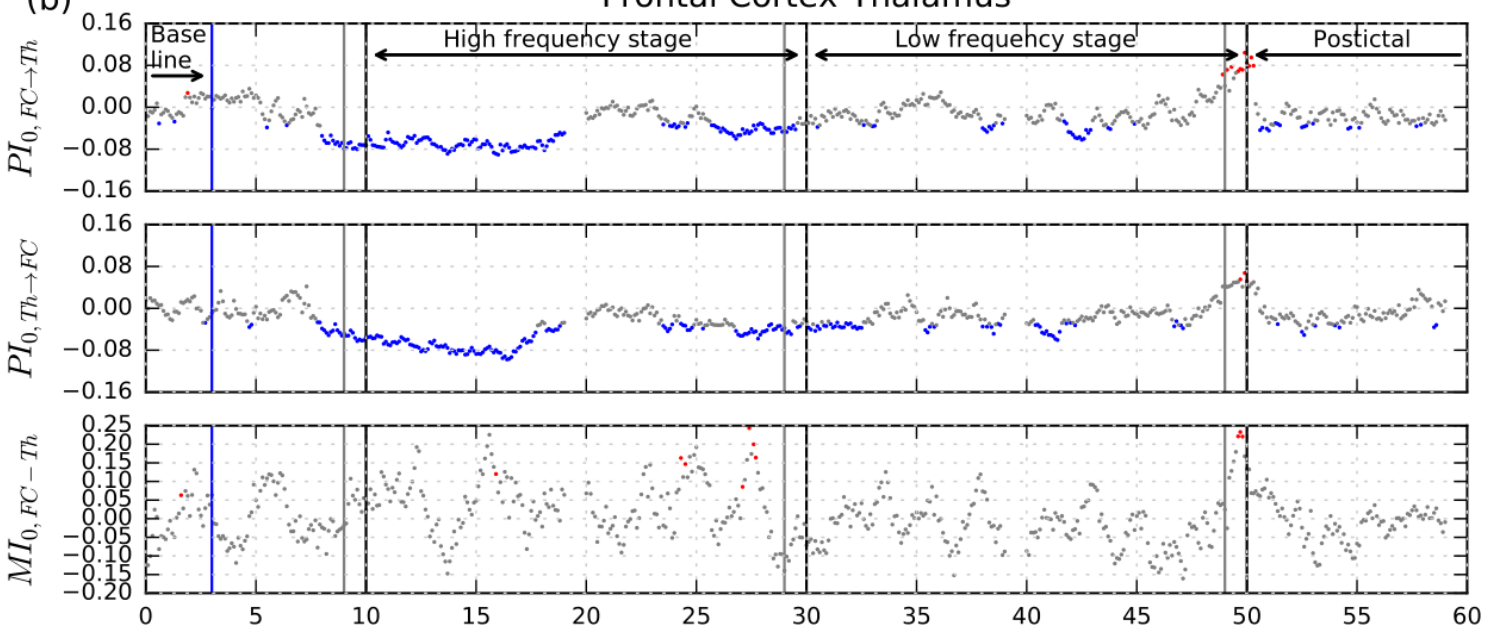

(c)

Frontal Cortex-Hippocampus
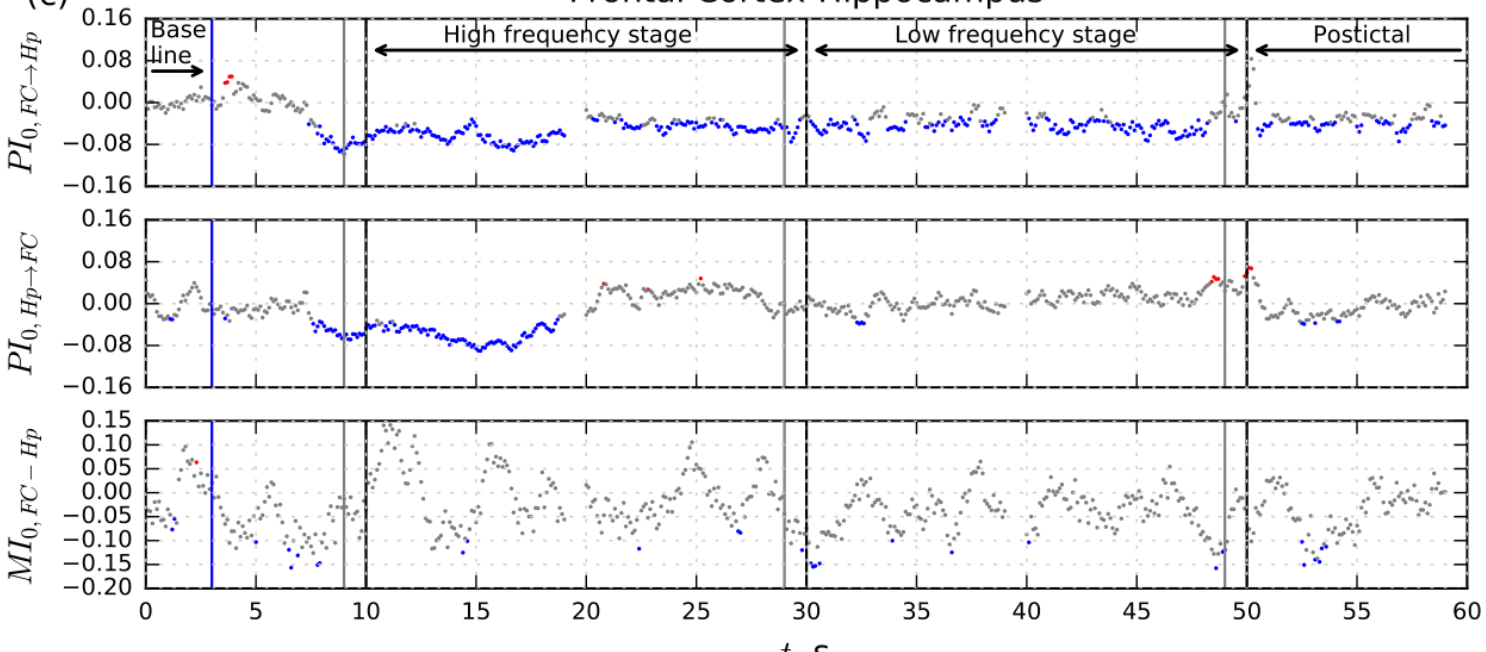

Figure 2. Dependencies of mean normalized prediction improvement $P I_{0}$ and mutual information function $\mathbf{M I _ { 0 }}$ on time for pair $\mathrm{FC}-\mathrm{MB}$, FC-Th, FC-Hp, calculated in $1 \mathrm{~s}$ time window. Red and blue dots indicate values significantly ( $<<0.05$ with Bonferroni correction) different (red - larger, blue - smaller) from baseline level, gray dots - not different. Black dashed vertical lines indicate seizure onset, transition to the second stage and termination, gray line before them - length of time window. Baseline, first (high frequency) stage, second (low frequency) stage and postictal period are subscribed on the top of each subfigure. 
(a)

Thalamus-Midbrain
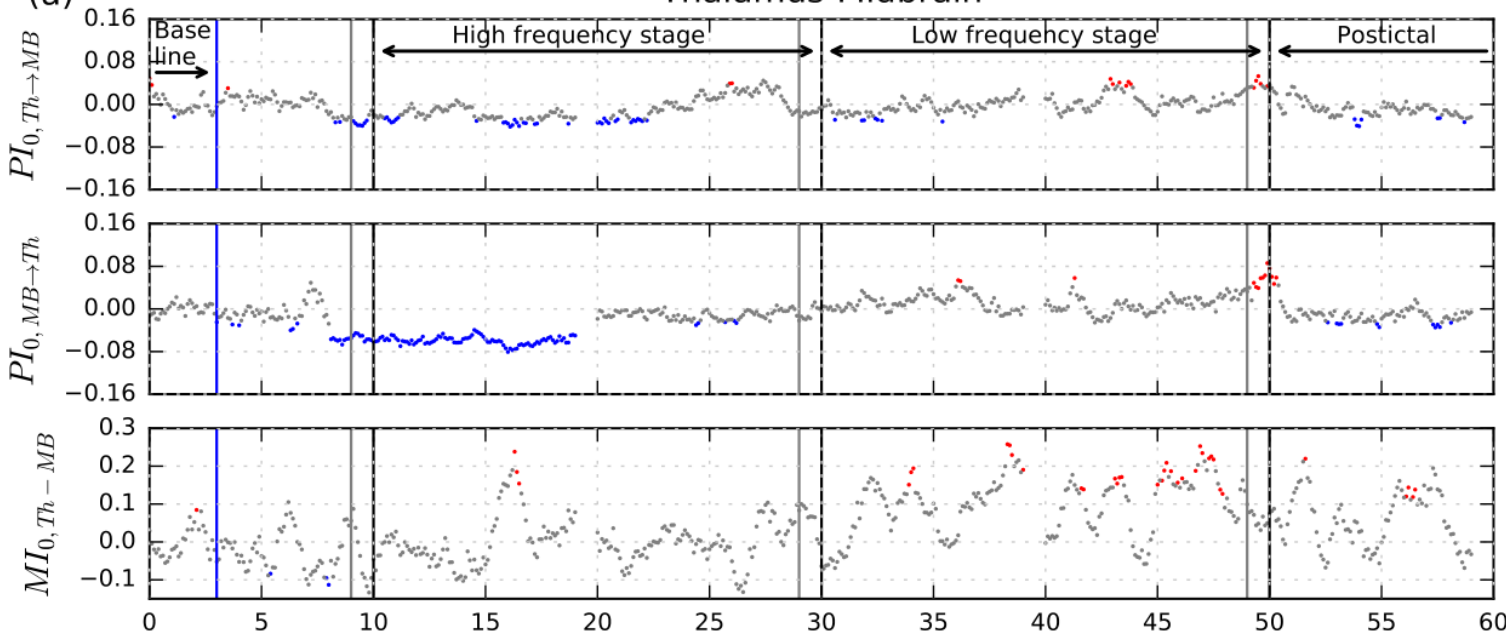

(b)

Hippocampus-Midbrain
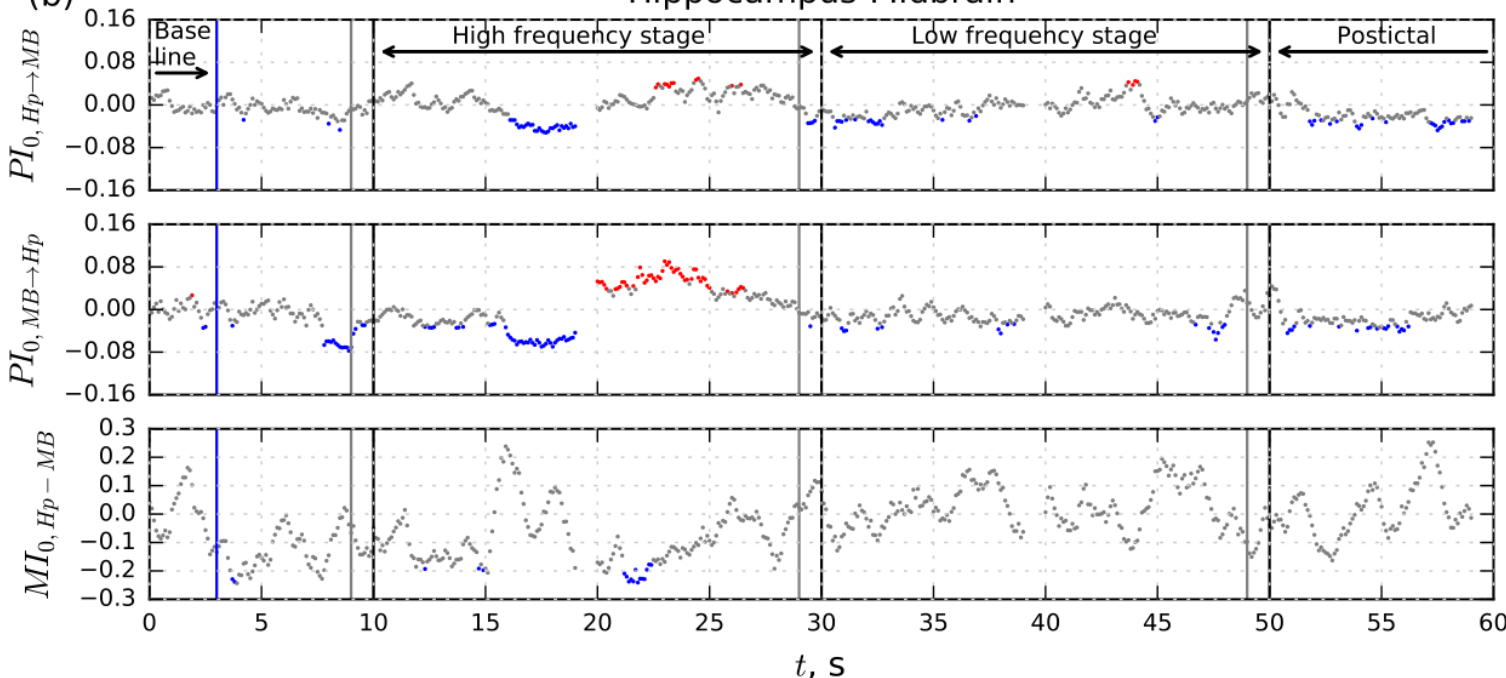

(c)

Hippocampus-Thalamus
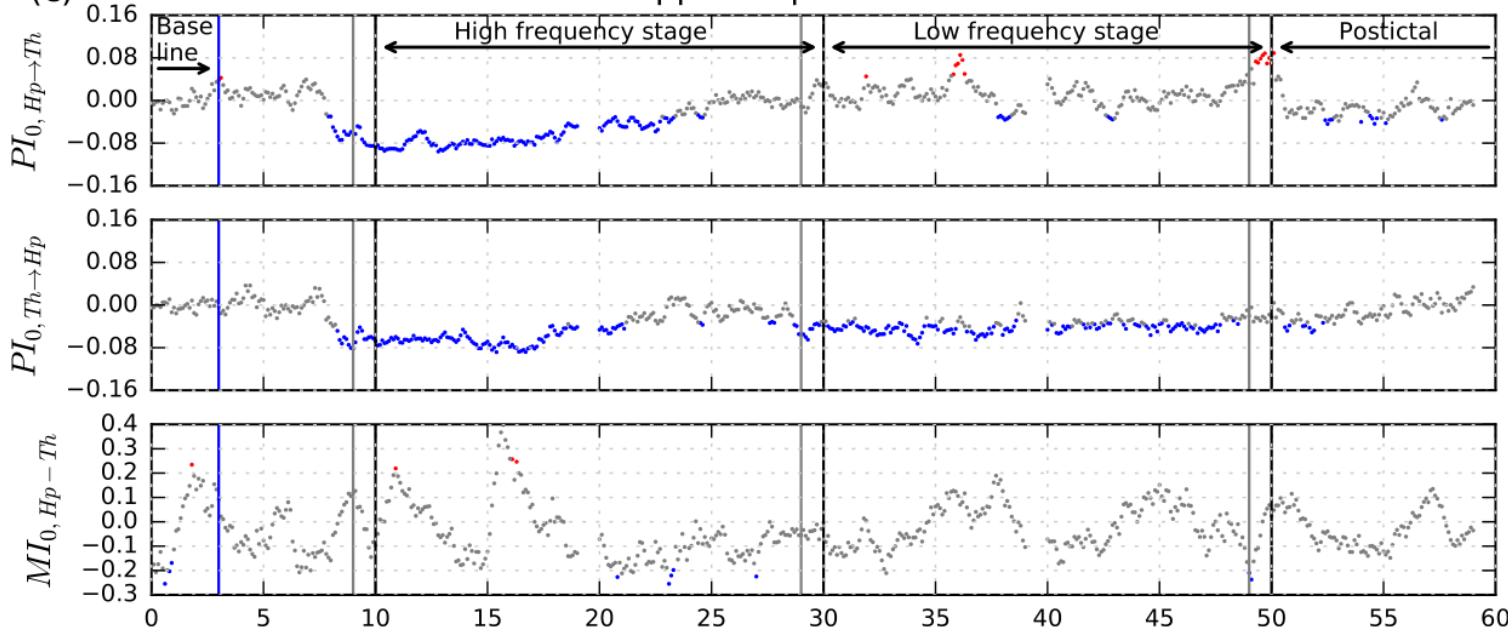

Figure 3. Dependencies of mean normalized prediction improvement $P I_{0}$ and mutual information function $M I_{0}$ on time for pair Th-MB, $\mathrm{Hp}-\mathrm{MB}$, $\mathrm{Hp}-\mathrm{Th}$, calculated in $1 \mathrm{~s}$ time window. Red and blue dots indicate values significantly ( $<<0.05$ with Bonferroni correction) different (red - larger, blue - smaller) from baseline level, gray dots - not different. Black dashed vertical lines indicate seizure onset, transition to the second stage and termination, gray line before them - length of time window. Baseline, first (high frequency) stage, second (low frequency) stage and postictal period are subscribed on the top of each subfigure. 

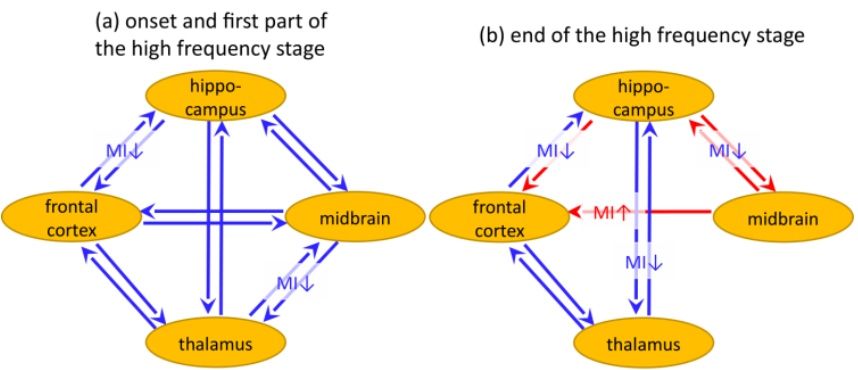

(c) first part of the low frequency stage

(d) end of the low frequency stage and seizure termination

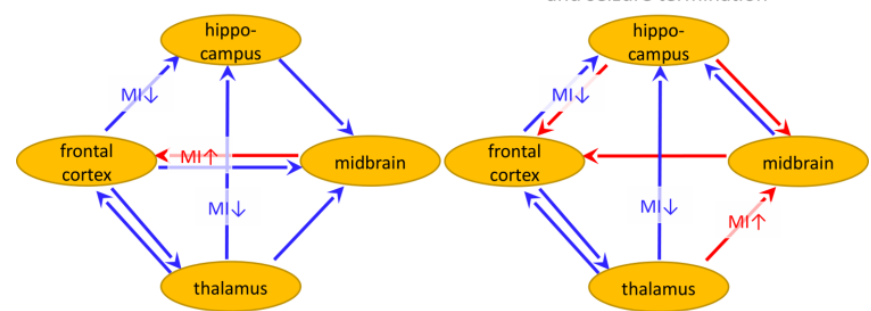

Figure 4. Schemes of significant changes in coupling at different stages of seizure. Blue/red arrows show significant decay/increase of prediction improvement $\boldsymbol{P I}_{0}$, significant changes in the mutual information function are shown near the arrows by signs MI $\uparrow$ (red) and MI $\downarrow$ (blue).

\subsubsection{Seizure initiation}

About 2 seconds before the start of a seizure, a drop in coupling is seen in all considered channel pairs. This decoupling continues during at least first 10 seconds of the high frequency stage (see Figure 2, blue dots) except hippocampus - midbrain pair, in which coupling restores to normal level at the seizure onset. A new bidirectional drop in coupling in this pair starts about 6 seconds after seizure onset.

\subsubsection{Transition to low frequency stage}

The driving from the FC to the HP remains lower than baseline during the whole seizure (Figure $2 c$ upper panel). For all other channel pairs the coupling restores in the course of the high frequency stage with some global transient increases: unidirectional from the $\mathrm{MB}$ and $\mathrm{HP}$ to the FC (Figure 2a and Figure $2 \mathrm{c}$ middle panels) and from the $\mathrm{TH}$ to the MB (Figure 3a upper panel), bidirectional in the HP-MB loop (Figure $3 \mathrm{~b}$ upper and middle panel).

Towards the transition from the high frequency stage to the low frequency one, all couplings are fairly equal to baseline except from for the FC-TH loop, which decreases about 5 seconds before the transition (Figure $2 \mathrm{~b}$, upper and middle panel), and except the above mentioned FC-HP loop, which shows decreased coupling during the whole seizure (Figure 2c, upper panel).

During the first few seconds of the low frequency stage a lower than baseline coupling is seen from FC and HP to MB (Figures $2 \mathrm{a}$ and $2 \mathrm{e}$, upper panels) and from $\mathrm{TH}$ to $\mathrm{FC}$ (Figure $2 \mathrm{~b}$, middle panel). The couplings from FC and TH to HP are also lower than baseline, but these decouplings last the whole low frequency stage (Figure 2c, upper panel, and Figure 3c, middle panel).

\subsubsection{Seizure termination}

Towards the end of the seizures, some global transient increased couplings are observed - the same increases which were observed towards the end of the high frequency stage: unidirectional from the MB and $\mathrm{HP}$ to the FC (Figure 2a and Figure $2 \mathrm{c}$ middle panels), and from the $\mathrm{TH}$ to the $\mathrm{MB}$ (Figure 3a upper panel), but the bidirectional increase in the $\mathrm{HP}-\mathrm{MB}$ loop present in the high frequency stage, is now only significant for the direction from the HP to the MB, (Figure $3 \mathrm{~b}$ upper panel). The MB to HP increase is absent, while this increase was very pronounced in the end of high frequency stage (Figure $3 \mathrm{~b}$ middle panel). Moreover, the drivings from FC and TH to HP are still low (Figure 2c, upper panel, and Figure 3c, middle panel).

When the moving window covers the seizure termination, there is a sharp increase in coupling in all pairs of channels in which the thalamus is involved as a driven structure (Figures $2 \mathrm{~b}, 3 \mathrm{a}$ and $3 c$ ). However, such an increase was mentioned previously as a method artifact [24]. In the postictal stage all couplings are generally rather low compared to baseline.

\subsection{Mutual information analysis}

Results of the mutual information analysis are shown in the lower panel of each subfigure. The curves of normalized mutual function $\mathrm{MIO}_{0}(t)$ vastly oscillate and most values are insignificant. Distributed along the whole seizure period, for some time points, the mutual information is significantly higher than baseline for the FC-MB pair (Figure 2a), while the $M l$ is smaller than the baseline for the FC-HP and TH-HP pairs (Figures $2 \mathrm{c}$ and $3 \mathrm{c}$ ). In the $5 \mathrm{~s}$ before the termination of the seizure, a number of values of $M I$ are significantly higher than baseline for the TH-MB pair (Figure 3a).

\section{Discussion}

The complete scheme of complex changes in the network was plotted in Figure 4. The main outcomes are formulated as follows:

i) During limbic seizures two stages were distinguished: the high frequency $(15-20 \mathrm{~Hz})$ stage, followed by the low frequency $(2 \mathrm{~Hz})$ stage;

ii) About 2 seconds before the seizure start, there is a drop in coupling in all considered channel pairs. This decoupling continues during at least first $10 \mathrm{~s}$ of the high frequency stage;

iii) During the whole seizure the driving from the frontal cortex to the hippocampus remains decreased. For all other channel pairs, the coupling restores during the high frequency stage with some global transient increases;

iv) In the postictal period all couplings are rather low compared to baseline.

\subsection{Discussion on Methods}

Results of coupling analysis from complex time series are always method dependent [22]. Simple methods, such as linear correlation and coherence function can be easily calculated using standard toolboxes such as MATLAB or SciPy. However, it is generally understood that these methods are not entirely adequate due to the following reasons: 1) only linear similarities can be analyzed, 2) coupling direction cannot be revealed, 3 ) the cause of similarity remains unknown (unidirectional driving, bidirectional one, common source, mediated driving through some intermediate structure, or simply a random coincidence of oscillations). Some nonlinear measures, like mutual information function [31], nonlinear 
correlation coefficient [35], and phase synchronization index [36] overcome the first problem, but not the others.

The mutual information function was already used for studying mechanisms of epilepsy in animals [37] and humans [38] before. The time shift between series at which the measure demonstrated the maximum was considered as a way to determine the coupling direction. However, recent studies clearly showed that undirected by nature nonlinear measures of similarity, including phase synchronization indeces [39] and mutual information [40] are unsafe for determining the coupling directionality, since very often the results occur to be random and misleading even for the relatively simple simulated series. In the current study, the mutual information function calculated using the most advantageous approach [31] was applied but with very limited success: a lot of results are either insignificant or cannot be linked with the results of the spectral analysis or Granger causality.

The most advantageous methods, allowing to address all three mentioned problems, are Granger causality [41], partial directed coherence [42], phase dynamics [43], and transfer entropy [33]. All these methods are more or less parametric. Generally, parametrization sufficiently decreases data requirements. In principle, it is possible to extract information about directed coupling from relatively short series: 30 oscillations for phase dynamics [43] and 4-8 oscillation for Granger causality [23]. However, the risk to obtain false positive (insufficient specificity) and false negative (insufficient sensitivity) results due to wrong parametrization also rises [25, 44, 45]. Till now, there is no possibility to obtain an absolute warranty against false results; one can only reduce risks by means of adaptation of used methods to specifics of studied data.

In the present study the pairwise analysis was preformed. It is not safe, since direct and indirect couplings cannot be completely separated. But, the classical conditional Granger causality [41] as well as more advanced approaches [46], which aim to eliminate the redundancy, cannot be applied directly, since they demand too many data. For example, in [46] 4,000 data points (10 s of recording, $400 \mathrm{~Hz}$ sampling frequency) were used with linear Granger causality, while to study limbic seizures one has to operate with nonlinear causality (so, much more coefficients have to be estimated), otherwise the method specificity becomes very low, as it was shown in refs 24,41 , and 40 . Also, relatively short epochs, not larger than $1 \mathrm{~s}$, have to be considered due to signal nonstationarity. However, the intracranially recorded local field potentials are much less redundant than surface EEGs, especially in the case if they were obtained from different and distinct brain structures as in the present study.

\subsection{Discussion on coupling changes during seizures}

The performed analysis was based on averaging over 30 seizure recorded in three SLV-treated rats (the whole data set from 3 animals). In [47] the large variability of results of statistical models constructed from EEGs was found. Therefore, in additiona to already reported results the results of Granger causality were also averaged independently for individual animals, and animal based results were found to follow the whole set based averaged results, but with less significance. Therefore, the conclusions were made based on the whole data set.

Reduced driving from the frontal neocortex to subcortical structures and increased driving from subcortical sites to the neocortex, described during generalized limbic seizures in the present study, are in line with the results of the imaging studies in both epileptic rats 48 and patients with TLE $[15,16]$. These studies have shown that limbic seizures are accompanied by activation of subcortical structures and neocortical deactivation. It has been hypothesized that abnormally increased activity of the thalamus and upper brainstem prevents normal activation of the cortex and leads to abnormally reduced function of the fronto-parietal cortex, which underlies abnormal motor behavior and impaired consciousness during complex partial seizures [14, 15]. Our results show that, despite involvement of the frontal cortex in the seizure expression, it plays only a passive role, being driven by subcortical structures.

The main active players seem to be the hippocampus and midbrain. Both structures show intrinsic epileptogenicity and contain triggering areas for certain seizure types: the hippocampus for limbic seizures, the midbrain for reflex audiogenic seizures [18, 19]. These two structures send direct and indirect glutamatergic projections to the cortex, thalamus and to each other. The hippocampus and midbrain may represent seizure drivers and their reciprocal excitatory interaction during the limbic seizures may represent a positive feedback loop driving the seizures.

While the central role of the hippocampus in TLE is well known, a role of the midbrain in limbic seizures is surprising. Nevertheless, participation of the midbrain in mechanisms of secondary generalized limbic seizures [49] and primary generalized tonic-clonic seizures [17] has been shown. It has been previously shown that chronic treatment with another CB1 receptor antagonist (rimonabant) significantly facilitates seizure spreading from the midbrain to cortex during audiogenic kindling [8]. It is interesting to investigate, whether the active participation of the midbrain in limbic seizures is a common phenomenon, or it is observed only during limbic seizures induced by cannabinoid antagonists.

\section{Conclusion}

The results of our study indicate that the cortico-thalamic loop, underlying the maintenance of absence seizures [50,51], is decoupled during the whole generalized limbic seizure, that corresponds to reduced thalamo-cortical functional connectivity in patients with TLE [20]. On the other hand, during limbic seizures the frontal cortex is driven by the hippocampus that is in contrast to cortico-hippocampal decoupling during cortico-thalamic absence seizures [52].

Conflict of interest: none declared.

\section{Funding}

This research was funded by Stipendium of President of Russian Federation for support of young scientists C Foundation for Basic Research, Grants No. 17-02-00307 and 18-015-00418.

\section{Ethical approval}

All applicable international, national, and institutional guidelines for the care and use of animals were followed.

\section{References}

1. Bertram EH. The functional anatomy of spontaneous seizures in a rat model of chronic limbic epilepsy. Epilepsia 1997; 38(1): 95-105. https://doi.org/10.1111/j.1528-1157.1997.tb01083.x. 
2. Spenser SS. Neural networks in human epilepsy: evidence of and implications for treatment. Epilepsia 2002; 43(3): 219-227. https://doi.org/10.1046/i.1528-1157.2002.26901.x.

3. Curia G, Longo D, Biagini G, Jones RSG, Avoli M. The pilocarpine model of temporal lobe epilepsy. J Neurosci Methods 2008; 172(2): 143-157. https://doi.org/10.1016/i.jneumeth.2008.04.019.

4. van Rijn CM, Perescis MFJ, Vinogradova L, van Luijtelaar G. The endocannabinoid system protects against cryptogenic seizures. Pharmacological Reports 2011; 63(1): 165-168. https://doi.org/10.1016/S1734-1140(11)70411-X.

5. Wallace MJ, Blair RE, Falenski KW, Martin BR DeLorenzo RJ. The endogenous cannabinoid system regulates seizure frequency and duration in a model of temporal lobe epilepsy. J Pharmacol Exp Ther 2003; 307(1): 129-137. https://doi.org/10.1124/jpet.103.051920.

6. Alger B. E.Endocannabinoids and their implication for epilepsy. Epilepsy Curr 2004; 4(5): 169-173. https://doi.org/10.1111/i.15357597.2004.04501.x.

7. Suleymanova EM, Shangaraeva VA, van Rijn CM, Vinogradova LV. The cannabinoid receptor agonist WIN55.212 reduces consequences of status epilepticus in rats. Neuroscience 2016; 334(): 191-200. https://doi.org/10.1016/j.neuroscience.2016.08.004.

8. Vinogradova LV, Shatskova AB, van Rijn CM. Pro-epileptic effects of the endocannabionoid receptor antagonist SR141716 in a model of audiogenic epilepsy. Epilepsy Research 2011; 96(3): 250-256. https://doi.org/10.1016/j.eplepsyres.2011.06.007.

9. Katona I, Freund TF. Multiple functions of endocannabinoid signaling in the brain. Ann Rev Neurosci 2012; 35: 529-558. https://doi.org/10.1146/annurev-neuro-062111-150420.

10. Lutz B. On-demand activation of the endocannabinoid system in the control of neuronal excitability and epileptiform seizures. Biochem Pharmacol 2004; 68(9): 1691-1698. https://doi.org/10.1016/i.bcp.2004.07.007.

11. Fernandez-Ruiz J, Gonzales S. Cannabinoid control of motor function at the basal ganglia. In: Handbook of Experimental Pharmacology. Cannabinoids. Springer, 2005: 479-507. https://doi.org/10.1007/3540-26573-2 16.

12. Herkenham M, Lynn AB, Little MD, Johnson MR, Melvin LS, de Costa BR, Rice KC. Cannabinoid receptor localization in brain. Proc Natl Acad Sci USA 1990; 87(5): 1932-1936. http://www.pnas.org/content/87/5/1932.

13. Monory K, Massa F, Egertová M, Eder M, Ruth HB. The endocannabinoid system controls key epileptogenic circuits in the hippocampus. Neuron 2006; 51(4): 455-466. https://doi.org/10.1016/j.neuron.2006.07.006.

14. Norden AD, Blumenfeld $\mathrm{H}$. The role of subcortical structures in human epilepsy. Epilepsy Behav 2002; 3(3): 219-231. https://doi.org/10.1016/S1525-5050(02)00029-X.

15. Blumenfeld H, Varghese GI, Purcaro MJ, Motelow JE, Enev M, McNally $\mathrm{KA}$, et al. Cortical and subcortical networks in human secondarily generalized tonic-clonic seizures. Brain 2009; 132(4): 999-1012. https://doi.org/10.1093/brain/awp028.

16. Haneef Z, Lenartowicz A, Yeh HJ, Levin HS, Engel J Jr, Stern JM. Functional connectivity of hippocampal networks in temporal lobe epilepsy. Epilepsia 2014; 55(1): 137-145. https://doi.org/10.1111/epi.12476.

17. Browning RA, Nelson DK. Modification of electroshock and pentylenetetrazol seizure patterns in rats after precollicular transections. Experimental Neurology 1986; 93(3): 546-556. https://doi.org/10.1016/0014-4886(86)90174-3.

18. Gale K. Subcortical structures and pathways involved in convulsive seizure generation. J Clin Neurophysiol 1992; 9(2): 264-277. https://www.ncbi.nlm.nih.gov/pubmed/1350593.

19. McCown TJ, Greenwood RS, Breese GR. Inferior collicular interactions with limbic seizure activity. Epilepsia 1987; 28(3): 234-241. https://doi.org/10.1111/j.1528-1157.1987.tb04213.x.
20. He X, Doucet GE, Sperling M, Sharan A, Tracy JI. Reduced thalamocortical functional connectivity in temporal lobe epilepsy. Epilepsia 2015; 56(10): 1571-1579. https://doi.org/10.1111/epi.13085.

21. Dinkelacker $V$, Valabregue $R$, Thivard L, Lehéricy $S$, Baulac $M$, Samson S, Dupont S. Hippocampal-thalamic wiring in medial temporal lobe epilepsy: Enhanced connectivity per hippocampal voxel. Epilepsia 2015; 56(8): 1217-1226. https://doi.org/10.1111/epi.13051.

22. Gourevitch B, Le Bouquin-Jeannes R, Faucon G. Linear and nonlinear causality between signals: methods, examples and neurophysiological applications. Biological Cybernetics 2006; 95(4): 349-369. https://doi.org/10.1007/s00422-006-0098-0.

23. Sysoeva MV, Sitnikova E, Sysoev IV, Bezruchko BP, van Luijtelaar G. Application of adaptive nonlinear Granger causality: Disclosing network changes before and after absence seizure onset in a genetic rat model. J Neurosci Methods 2014; 226: 33-41. https://doi.org/10.1016/j.jneumeth.2014.01.028.

24. Sysoev IV, Sysoeva MV. Detecting changes in coupling with Granger causality method from time series with fast transient processes. Physica D: Nonlinear Phenomena 2015; 309: 9-19. https://doi.org/10.1016/i.physd.2015.07.005.

25. Kornilov MV, Medvedeva TM, Bezruchko BP, Sysoev IV. Choosing the optimal model parameters for Granger causality in application to time series with main timescale. Chaos, Solitons \& Fractals 2016; 82: 11-21. https://doi.org/10.1016/j.chaos.2015.10.027.

26. Perescis MFJ, de Bruin N, Heijink L, Kruse C, Vinogradova L, Lüttjohann A, et al. Cannabinoid antagonist SLV326 induces convulsive seizures and changes in the interictal EEG in rats. PLOS ONE 2017; 12(2): e0165363. https://doi.org/10.1371/journal.pone.0165363.

27. Smirnov DA, Mokhov II. From Granger causality to long-term causality: Application to climatic data. Phys Rev E 2009; 80: 016208. https://doi.org/10.1103/PhysRevE.80.016208.

28. Hesse W, Möller E, Arnold M, Schack B. The use of time-variant EEG Granger causality for inspecting directed interdependencies of neural assemblies. J Neurosci Methods 2003; 124(1): 27-44. https://doi.org/10.1016/S0165-0270(02)00366-7.

29. Sysoeva MV, Dikanev TV, Sysoev IV. Selecting time scales for empirical model construction. Izvestiya VUZ, Applied Nonlinear Dynamics 2012; 20(2): 54-62. https://doi.org/10.18500/0869-6632-2012-20-2-54-62.

30. Kornilov MV, Sysoev IV. Investigating nonlinear Granger causality method efficiency at strong synchronization of systems. Izvestiya VUZ, Applied Nonlinear Dynamics 2014; 22(4): 66-76. https://doi.org/10.18500/0869-6632-2014-22-4-66-76.

31. Kraskov A, Stögbauer H, Grassberger P. Estimating mutual information. Phys Rev E 2004; 066138. https://doi.org/10.1103/PhysRevE.69.066138.

32. Kugiumtzis D. Direct-coupling information measure from nonuniform embedding. Phys Rev E 2013; 87: 062918. https://doi.org/10.1103/PhysRevE.87.062918.

33. Schreiber T. Measuring information transfer. Phys Rev Lett 2000; 85: 461. https://doi.org/10.1103/PhysRevLett.85.461.

34. Montalto A, Faes L, Marinazzo D. MuTE: a MATLAB toolbox to compare established and novel estimators of the multivariate transfer entropy. PLOS One 2014; 9(10): e109462. https://doi.org/10.1371/journal.pone.0109462.

35. Pijn JPM, Vijn PCM, Lopes da Silva FH, Van Ende Boas W, Blanes W. Localization of epileptogenic foci using a new signal analytical approach. Neurophysiol Clin 1990; 20: 1-11. https://doi.org/10.1016/S0987-7053(05)80165-0.

36. Allefeld C. Kurths J. Testing for phase synchronization. Int J Bif Chaos 2004; 14(2): 405-416. https://doi.org/10.1142/S021812740400951X2.

37. Mars NJI, Lopes da Silve FH. Propagation of seizure activity in kindled dogs. Electroencephalography and clinical neurophysiology 1983; 56(2): 194-209. https://doi.org/10.1016/0013-4694(83)90074-3.

38. Mars NJI, Thompson PM, Wilkus RJ. Spread of epileptic seizure activity in humans. Epilepsia 1985; 26(1): 85-94. https://doi.org/10.1111/j.1528-1157.1985.tb05192.x. 
39. Vakorin VA, Mišić B, Krakovska O, Bezgin G, Mclntosh R. Confounding effects of phase delays on causality estimation. PLoS One 2013; 8(1): e53588. https://doi.org/10.1371/journal.pone.0053588.

40. Smirnov DA. Quantifying causal couplings via dynamical effects: a unifying perspective. Phys Rev E 2014; 90: 062921. https://doi.org/10.1103/PhysRevE.90.062921.

41. Chen YH, Rangarajan G, Feng JF, Ding MZ. Analyzing multiple nonlinear time series with extended Granger causality. Phys Lett $A$ 2004; 324(1): 26-35. https://doi.org/10.1016/i.physleta.2004.02.032.

42. Schelter B, Timmer J, Eichler M. Assessing the strength of directed influences among neural signals using renormalized partial directed coherence. J Neurosci Methods 2009; 179(1): 121-130. https://doi.org/10.1016/i.jneumeth.2009.01.006.

43. Smirnov DA, Bezruchko BP. Estimation of interaction strength and direction from short and noisy time series. Phys Rev E 2003; 68: 046209. https://doi.org/10.1103/PhysRevE.68.046209.

44. Smirnov DA, Bezruchko BP. Spurious causalities due to low temporal resolution: Towards detection of bidirectional coupling from time series. Europhys Lett 2012; 100(1): 10005. https://doi.org/10.1209/0295-5075/100/10005.

45. Smirnov DA. Spurious causalities with transfer entropy. Phys Rev $E$ 2013; 87: 042917. https://doi.org/10.1103/PhysRevE.87.042917.

46. Stramaglia S, Cortes JM, Marinazzo D. Synergy and redundancy in the Granger causal analysis of dynamical networks. New Journal of Physics 2014; 16(10): $105003 . \quad$ https://doi.org/10.1088/13672630/16/10/105003.

47. Papadopoulou M, Cooray G, Rosch R, Moran R, Marinazzo D, Friston K. Dynamic causal modelling of seizure activity in a rat model. Neuroimage 2017; 146: 518-532. https://doi.org/10.1016/j.neuroimage.2016.08.062.

48. Englot DJ, Modi B, Mishra AM, DeSalvo M, Hyder F, Blumenfeld H. Cortical deactivation induced by subcortical network dysfunction in limbic seizures. J Neurosci 2009; 29(41): 13006-13018. https://doi.org/10.1523/JNEUROSCI.3846-09.2009.

49. Chiba S, Wada JA. Amygdala kindling in rats with brainstem bisection. Brain Research 1995; 682(1-2): 50-54. https://doi.org/10.1016/00068993(95)00315-H.

50. Meeren HK, Veening JG, Möderscheim TA, Coenen AM, van Luijtelaar G. Thalamic lesions in a genetic rat model of absence epilepsy: dissociation between spike-wave discharges and sleep spindles. Exp Neurol 2009; 217(1): 25-37. https://doi.org/10.1016/j.expneurol.2009.01.009.

51. Stefan H, Lopes da Silva FH. Epileptic neuronal networks: methods of identification and clinical relevance. Front Neurol 2013; 4: 8. https://doi.org/10.3389/fneur.2013.00008.

52. Sysoeva MV, Vinogradova LV, Kuznetsova GD, Sysoev IV, van Rijn CM. Changes in corticocortical and corticohippocampal network during absence seizures in WAG/Rij rats revealed with time varying Granger causality. Epilepsy Behav 2016; 64(Pt A): 44-50. https://doi.org/10.1016/i.yebeh.2016.08.009.

\section{Authors:}

Ilya V. Sysoev - PhD, Assistant Professor, Department of Dynamic Modeling and Biomedical Engineering, Saratov State University, Saratov, Russia; Senior Researcher, Laboratory of Modelling in Nonlinear Dynamics, Saratov Branch of the Institute of RadioEngineering and Electronics of Russian Academy of Sciences, Saratov, Russia. http://orcid.org/0000-00029203-5504.

Martin F.J. Perescis - PhD student, Donders Centre for Cognition, Radboud University, Nijmegen, Netherlands; Lecturer, HAS University Of Applied Sciences, 's-Hertogenbosch, Netherlands. http://orcid.org/0000-00015540-4983.

Lyudmila V. Vinogradova - DSc, Leading Researcher, Institute of Higher Nervous Activity and Neurophysiology, Moscow, Russia. http://orcid.org/0000-0002-3471-7240.

Marina V. Sysoeva - PhD, Assistant Professor, Yuri Gagarin State Technical University of Saratov, Saratov, Russia. http://orcid.org/0000-0003-4228$\underline{9124}$
Clementina M. van Rijn - PhD, Docent, Donders Centre for Cognition, Radboud University, Nijmegen, Netherlands. 